\title{
Activity and Mode of Action of Flavonoids Compounds Against Intracellu- lar and Extracellular Forms of Trypanosoma cruzi
}

\author{
Samira Boutaleb-Charki ${ }^{1}$, Manuel Sánchez-Moreno ${ }^{1}$, Jesús G. Díaz ${ }^{2}$, María J. Rosales ${ }^{1}$, \\ Oscar Huertas ${ }^{1}$, Ramon Gutierrez-Sánchez ${ }^{3}$ and Clotilde Marín*,1 \\ ${ }^{1}$ Department of Parasitology, University of Granada, Severo Ochoa s/n, E-18071 Granada, Spain \\ ${ }^{2}$ Department of Chemistry, University of La Laguna, Instituto Universitario de BioOrgánica "Antonio González", \\ E-38206, La Laguna, Tenerife, Spain \\ ${ }^{3}$ Department of Statistics, University of Granada, Severo Ochoa s/n, E-18071 Granada, Spain
}

\begin{abstract}
This study investigates the in vitro activity of a number of flavonoids (kaempferol, quercetin, trifolin, and acetyl hyperoside) and their acetylated products: kaempferol acetate, quercetin acetate, trifolin acetate, and acetyl hyperoside acetate) isolated from the aerial parts of plant Consolida oliveriana against epimastigote, amastigote and metacyclic forms of $T$. cruzi, their cytotoxic against a host Vero-cell line and analyse the possible mechanism by which these molecules act. Acetylated compounds were potent $T$. cruzi epimastigote growth inhibitors with activity levels similar to those of benznidazole, used as the reference drug. These compounds, at the dosage $\mathrm{IC}_{25}$, decreased the ability of metacyclic forms to invade mammalian cells, their intracellular replications and transformation in trypomastigotes, with no toxicity to the host cells. The cells treated presented severe damage in their ultrastructure: intense vacuolization, and appearance of lysosomes as well as other residual bodies. The mitochondrial section appeared larger in size, with a swollen matrix. In addition, these compounds changed the excretion of end metabolites, primarily affecting acetate and succinate excretion, possibly by directly influencing certain enzymes or their synthesis. The potent tripanocidal activities of the flavons described here represent an exciting advance in the search for new antiprotozoal agents.
\end{abstract}

Keywords: Flavonoids compounds, Consolida oliveriana, antitripanocidal agents, mode of action.

\section{INTRODUCTION}

Chagas disease is a complex zoonosis caused by Trypanosoma cruzi. It constitutes a permanent threat for almost a fourth of the total population of Latin America, estimated at 28 million people in the year 2006, which are under risk of infection, taking into account the geographic distribution of the vector insects and the multiple reservoirs involved in the diverse transmission cycles. The disease is found in Mexico as well as all of Central and South America, where the clinical manifestations and epidemiological characteristics are highly variable in the different zones where the disease is endemic [1].

It is important to take into account that migratory movements from rural to urban zones has changed the epidemiological characteristics of Chagas disease. It is estimated that around $70 \%$ of the Latin American population lives in urban areas, whereas the figures from the 1930 s showed $70 \%$ in rural areas. Thus, the infection that had been primarily rural has become urban and transmissible by blood transfusion. Furthermore, transmission is not limited to the countries in which the disease is endemic. In fact, it has extended to other countries such as Canada, the United States, and Spain, where cases of transmission of T. cruzi through blood products have been documented [2].

*Address correspondence to this author at the Department of Parasitology, University of Granada, Severo Ochoa s/n, E-18071 Granada, Spain; Tel: +34 958 242369; Fax: +34 958 243174; E-mail: cmaris@ugr.es
Drugs currently used to treat Chagas disease are nitroheterocyclic nitrofurane compounds: nifurtimox (Lampit $^{\circledR}$ Bayer) and benznidazole, a derivative of nitroimidazol (Rochagan $^{\circledR}$, Radanil ${ }^{\circledR}$, Roche), the anti-T. cruzi activities of which were empirically described three decades ago [3]. Nifurtimox and benznidazol show strong activity, which has been well documented in the acute phase (c. $80 \%$ cures in patients treated). However, in the indeterminate phase and in the chronic form, the results are less conclusive. Also, there is information indicating that the effectiveness of these drugs varies according to the geographical area, probably due to differences in the drug susceptibility of different $T$. cruzi strains. In addition, the common side-effects, including anorexia, vomiting, peripheral polyeuropathy, and allergic dermopathy, can in some cases require the interruption of the treatment [4].

The reasons for the great differences in the anti-parasite effectiveness of the nitro-heterocyclic compounds in the acute and chronic phases is not yet clear, but may be related to inadequate pharmocokinetic properties of the drugs in treating chronic infections. This has led to the conclusion that new drugs with a different pharmacological profile need to be developed.

One strategy to discover new drug is to investigate natural products from plants used medicina1ly [5]. Consolida, a highly specialized genus of Ranunculaceae closely allied to Delphinium with its centre of diversity in Anatolia, is, like its relatives, a rich source of alkaloids. Previous studies of the 
aerial parts of Consolida oliveriana (DC) Schrod., a species used medicinally in parts of Anatolia, have concentrated on the isolation of its constituent alkaloids [6]. Many publications have dealt with the diterpenoid alkaloids of other Consolida species but reports on the flavonoid content of members of this genus are scarse [7]. The biological action of those compounds is less known. There are a few reports on their plant defensive and pharmacological properties, including their effects on $T$. cruzi epimastigote forms and antileishmanial properties, but their neurotoxic effects are unknown $[8,9]$.

As part of the ongoing research by the Tenerife group on flavonoids in the genus Delphinium and related genera, we have investigated the most polar fraction of the ethanol extract of the aerial parts of $C$. oliveriana. In our study, we investigate the activity of a number of these flavonoids against epimastigote, amastigote, and metacyclic forms of $T$. cruzi. In addition, we examine the cytotoxic effects of these compounds against a host Vero-cell line and we analyse the possible mechanism by which these molecules act.

\section{MATERIALS AND METHODOLOGY}

\section{Parasite Strain, Culture and In Vitro Studies}

The Maracay strain of $T$. cruzi was isolated at the Institute of Malariology and Environmental Health in Maracay (Venezuela). Epimastigote forms were obtained in biphasic blood-agar NNN medium (Novy-Nicolle-McNea1) supplemented with MEM and $20 \%$ inactivated fetal bovine serum and afterwards reseeded in a monophasic culture (MTL), following the method of [10].

\section{Plant Material}

Aerial parts of the plant Consolida oliveriana (DC) Schrod. were collected and processed as done elsewhere [11]. The compounds obtained (Fig. 1) were dissolved in dimethyl sulfoxide (Panreac, Barcelona, Spain) at a concentration of $0.1 \%$, after being assayed as nontoxic and without inhibitory effects on the parasite growth, according to existing methods [10]. The compounds were dissolved in the culture medium, and the dosages used were: 100, 50, 25, 10 and $1 \mu \mathrm{M}$. The effect of each compound against epimastigote forms, as well as the concentrations, was evaluated at 24, 48
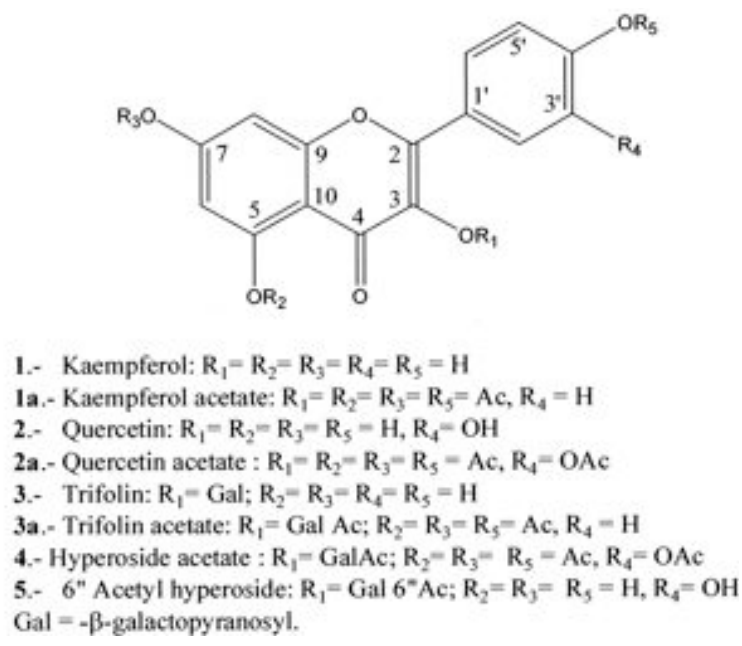

Fig. (1). Chemical structure of flavonoids compounds. and $72 \mathrm{~h}$ using a Neubauer hemocytometric chamber and the trypanocidal effect is expressed as $\mathrm{IC}_{50}$ values, i.e the concentration required to give $50 \%$ inhibition, calculated by linear regression analysis from the Kc values at the concentrations employed.

\section{Cell Culture and Cytotoxicity Tests}

Vero cells (F1ow) were grown in Minimal Essential Medium (MEM; Gibco) supplemented with $10 \%$ inactivated fetal calf serum and adjusted to $\mathrm{pH} 7.2$, in a humidified $95 \%$ air- $5 \% \mathrm{CO}_{2}$ atmosphere at $37^{\circ} \mathrm{C}$ for 2 days. The test for cytotoxicity, cells were placed in $25 \mathrm{ml}$ colie-based bottles (Sterling), and centrifuged at $100 \times \mathrm{g}$ for $5 \mathrm{~min}$. The culture medium was removed, and fresh medium was added to a final concentration of $1 \times 10^{5}$ cells $/ \mathrm{ml}$. This cell suspension was distributed in a culture tray (with 24 wells) at a rate of 100 $\mu \mathrm{l} /$ well and incubated for 2 days at $37^{\circ} \mathrm{C}$ in humid atmosphere enriched with $5 \% \mathrm{CO}_{2}$. The medium was removed, and the fresh medium was added together with the product to be studied (at a concentration 100, 50, 25, 10 and $1 \mu \mathrm{M}$ ). The cultures were incubated for $72 \mathrm{~h}$. The vital stain trypan blue $(0.1 \%$ in phosphate buffer) was used to determine cell viability. The number of dead cells was recorded, and the percent viability was calculated in comparison to that of the control culture, and the $\mathrm{IC}_{50}$ calculated by linear regression analysis from the Kc values at the concentrations employed.

\section{Transformation of Epimastigote to Metacyclic Forms}

To induce metacyclogenesis, parasites were cu1tured at $28^{\circ} \mathrm{C}$ in modified Grace's medium (Gibco) for 12 days according to the methods described by [12]. Twelve days after cultivation at $28^{\circ} \mathrm{C}$, metacyclic forms were counted in order to infect Vero cells. The proportion of metacyc1ic forms was around $40 \%$ at this stage.

\section{Amastigote- Vero Cell Assay}

Vero cells were cultivated in MEM medium in a humidified $95 \%$ air- $5 \% \mathrm{CO}_{2}$ atmosphere at $37^{\circ} \mathrm{C}$. Cells were seeded at a density of $1 \times 10^{5}$ cells/ well in 24-well microplates (Nunc) with rounded coverslips on the bottom and cultivated for 2 days. After were infected in vitro with T. cruzi metacyclic forms at a ratio of 10:1. The drugs ( $\mathrm{IC}_{25}$ concentrations) were added immediately after infection, and were incubated for $6 \mathrm{~h}$ at $37^{\circ} \mathrm{C}$ in a $5 \% \mathrm{CO}_{2}$. The non-phagocytosed parasites and the drugs were removed by washing and then the infected cultures were grown for 8 days in fresh medium. Fresh culture medium was added every $48 \mathrm{~h}$.

The drug activity was determined from the percentage of infected cells and the number of amastigotes per Vero cells infected in treated and untreated cultures in methanol-fixed and Giemsa-stained preparations. The percentage of infected Vero cells and the mean number of amastigotes per infected cell were determined by analyzing more than 100 host cells distributed in randomly chosen microscopic fields. Values are the means of four separate determinations. The number of trypomastigotes in the medium was determined as described previously [13].

\section{Ultrastructural Alterations}

The parasites, at a density of $5 \times 10^{6}$ cells $/ \mathrm{mL}$, were cultured in their corresponding medium, containing the drugs at la concentración de $\mathrm{IC}_{25}$. After $72 \mathrm{~h}$, the cultures were centri- 
fuged at $400 \mathrm{x} \mathrm{g}$ for $10 \mathrm{~min}$, and the pellets washed in PBS and then fixed with $2 \%(\mathrm{v} / \mathrm{v}) p$-formaldehyde-glutaraldehyde in $0.05 \mathrm{M}$ cacodylate buffer $(\mathrm{pH} 7.4)$ for $2 \mathrm{~h}$ at $4{ }^{\circ} \mathrm{C}$. Pellets were prepared for transmission-electron microscopy following the technique of [10].

\section{Metabolite Excretion}

Cu1tures of epimastigote forms of $T$ cruzi (initial concentration $5 \times 10^{5}$ cells $/ \mathrm{ml}$ ) received $\mathrm{de} \mathrm{IC}_{25}$ of the flavonoids conpounds (except for control cultures). After incubation for $72 \mathrm{~h}$ at $28^{\circ} \mathrm{C}$, the cells were centrifuged at $400 \mathrm{x} \mathrm{g}$ for 10 min. The supernatants were collected to determine excreted metabolites by nuclear magnetic resonance spectroscopy $\left({ }^{1} \mathrm{H}-\mathrm{NMR}\right)$ as previously described by [14]. The chemical displacements were expressed in parts per million (ppm), using sodium 2,2dimethyl-2-silapentane-5-su1fonate as the reference signal. The chemical displacements used to identify the respective metabolites were consistent with those described by [14].

\section{RESULTS AND DISCUSSION}

Plant-derived active principles and their semi-synthetic and synthetic analogues have served as a major route to new chemotherapy compounds [15,16]. Current conventional chemotherapy treatments are very expensive, toxic, and less effective in treating the disease. The use of natural products for the treatment of protozoal infections (Leishmania spp. and T. cruzi) is well known and has been documented since ancient times [5,9]. Flavonoids are found in abundance in diets rich in fruits, vegetables and plant-derived beverages and appear to have anti-cancer, anti-microbial and antiparasitic properties [11]. Here, we evaluate the antiprotozoan properties of a number of flavonoids obtained from natural sources and their acetylated derivatives.

The inhibitory effect of eight flavonoids compounds on the in vitro growth of $T$. cruzi epimastigotes was measured at different times following established procedures (see Materials and Methods). The results are displayed in Table $\mathbf{1}$ for benznidazole used as the reference drug and including toxicity values against Vero cells. The control epimastigote cells were cultured in the presence of dimethyl sulfoxide at the same concentration as that added to the cultures with the flavonoid compounds. Of the eight compounds assayed, four $(1,2,3$ and 5$)$ were obtained in a natural way from the plant Consolida oliveriana and the other four by acetylation of these natural products (1a, 2a, 3a and 4) and these latter four acetylated compounds proved the most active against $T$. cruzi epimastigotes. After $72 \mathrm{~h}$ of exposure, the compounds 1a y 4 showed $\mathrm{IC}_{50}$ values of 9.87 and $13.22 \mu \mathrm{M}$, respectively, close to those of benznidazole (referente drugs). These four acetylated compounds tested proved much less toxic than benznidazole against Vero cells. It bears mentioning that compounds $\mathbf{1 a}$ and $\mathbf{4}$ exhibited an inhibitory concentration of 102.5 and $188.5 \mu \mathrm{M}$ after $72 \mathrm{~h}$ of culture, respectively. This is 8 and 14 times higher than the corresponding value measured for benznidazole $(13.6 \mu \mathrm{M})$, respectively. The acetylation of certain flavonoids has been shown to increase the antiproliferative activities of the parent compounds against HL-60 and other cell lines. For example, 3a caused cell death in human leukaemia cells [15]. This greater effectiveness may be due to acetylation facilitating absorption of the product.
In most studies on activity assays of new compounds against parasites, forms that develop in vectors are used (epimastigote forms in the case of $T$. cruzi) [10], for the ease of working with these forms in vitro; however, in this study, we have included the effect of these compounds on the forms that are developed in the host (amastigotes and trypomastigotes), the study of which is of great importance, given that the final aim is to determine the effects in the definitive host. For this type of work and studies on the action mechanism, we selected the products that had the greatest inhibitory effect on the in vitro growth of $T$. cruzi (1a, 2a, 3a and 4) and that at the same time had less toxic effect on Vero cells, using the $\mathrm{IC}_{25}$ of each product as the test dosage.

Fig. (2A) illustrates $T$. cruzi propagation in Vero cell (with and without co-addition of the 1a, 2a, 3a and 4 compounds). When $1 \times 10^{5}$ Vero cells were incubated for 2 days and then infected with $1 \times 10^{6}$ metacyclic forms, the parasites invaded the cells and underwent morphologic conversión to amastigotes within 1 day after infection. On days 4, 6, 8 and 10 , the rate of host-cell infection increased to $15,46,80$ and $90 \%$, respectively. When flavonoids compounds (1a, 2a, 3a and 4) were added simultaneously to the infection of Vero cells with $T$. cruzi metacyclic forms ( $\mathrm{IC}_{25}$ concentration), the infection rate significantly decreased with respect to the control, reaching a 32 and $38 \%$ for the compounds $\mathbf{1 a}$ and 3a, respectively on day 10 . In the control experiments, the average number of amastigotes per infected cell increased to 85.6 on day 6, descreasing to 50 on day 10 (Fig. 2B). The compounds $4,1 \mathbf{a}$ and $\mathbf{3 a}$ inhibited $T$. cruzi amastigote replication in Vero cells in vitro. Thus, the addition of a concentration equivalent to the $\mathrm{IC}_{25}$ of these flavonoids compounds produces a markedly lowered the amastigote number per infected cell, reaching 60,56 y $42 \%$, respectively, reduction in amastigote number respect to control for cultures day 10 .

The decrease in the average amastigote number on day 7 for the control experiment coincided with the increase in trypomastigote numbers in the medium. This behavior is due to the rupture of the Vero cells with the subsequent release of amastigotes and futre transformation into trypomastigotes. The number of trypomastigotes in the medium was $1.8 \times 10^{6}$ parasites on day 10 . While, the three acetilated flavonoids compounds (4, 1a and 3a) gave 88, 71 and $59 \%$ reduction in trypomastigote numbers, respectively (Fig. 2C).

Alterations in the excretion of different metabolites, as well as inhibition of enzyme activity in the major metabolic pathways by which these organisms gain energy, are data of great use for elucidating the toxic activity in this field. The epimastigote forms of $T$. cruzi depend entirely on glycolysis for obtaining energy, as also happens in other trypanosomatids. The special compartmentalization presented by these organisms in the glycolytic pathway enables them to direct this process more efficiently than can a conventional eukaryotic cell [17]. The ability to utilize a fast and effective form of sugar is of unquestionable value in adapting and colonizing different hosts, especially given that these organisms lack a conventional system to store carbohydrates [18]. As far as it is known to date, none of the trypanosomatids studied can completely degrade glucose to $\mathrm{CO}_{2}$ under aerobic conditions, excreting into the medium as fermented metabolites a great part of their carbon skeleton, which differs depending on the species considered [19]. Trypanosoma cruzi 
consumes glucose at a high rate, thereby acidifying the culture medium due to incomplete oxidation to acids.
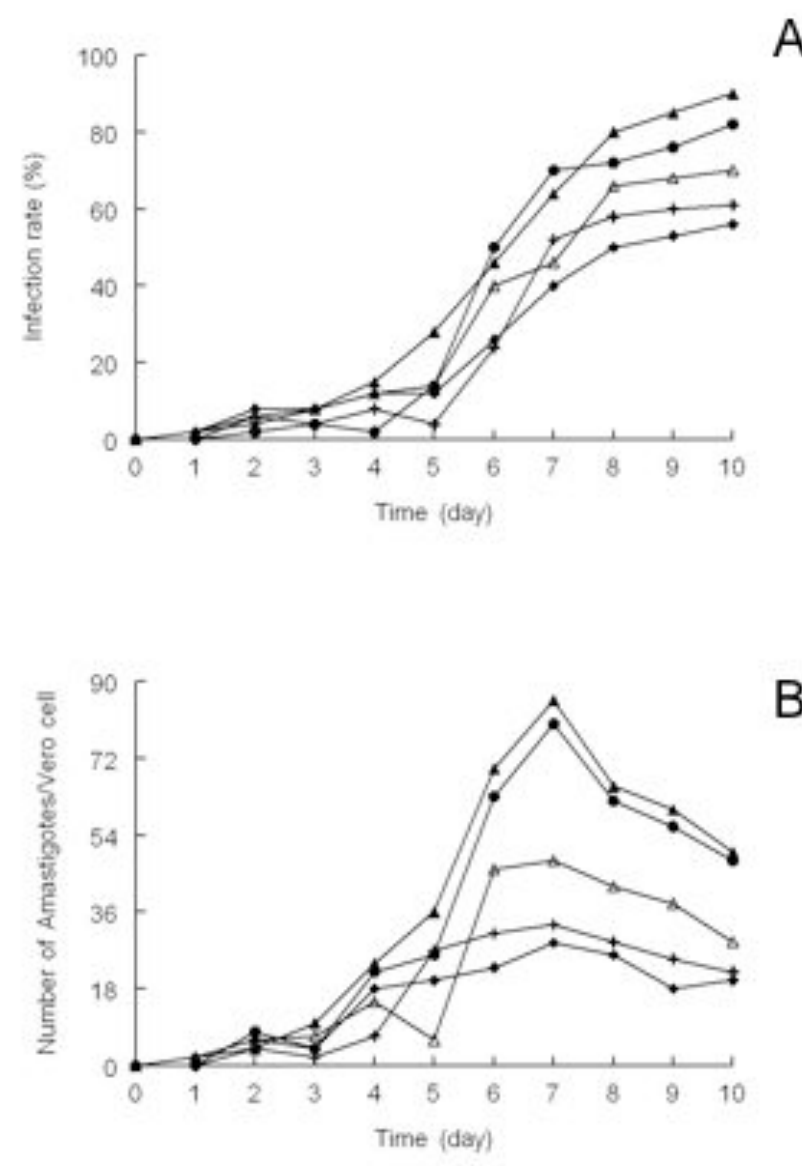

B

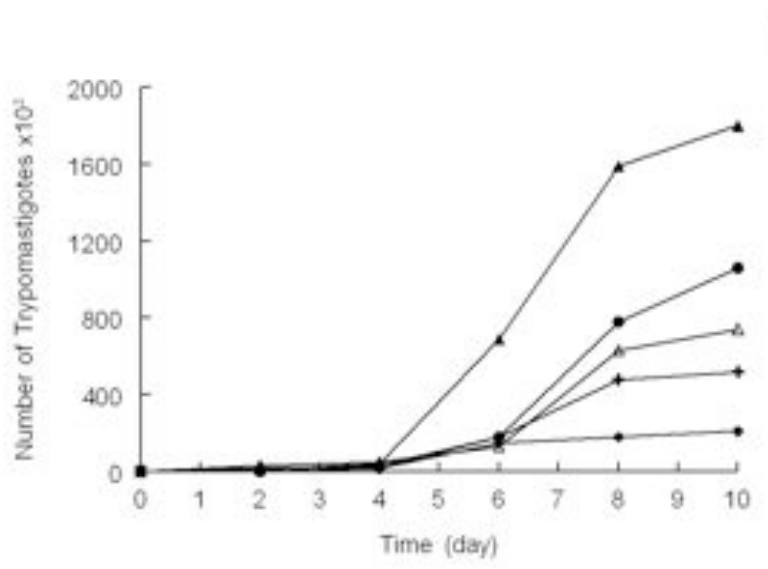

Fig. (2). Effect of acetylated flavonoids compounds on the infection rate and T. cruzi growth. (A) rate of infection. (B) mean number of amastigotes per infected Vero cell. (C) Number of trypomastigotes in the culture medium. - $\Delta$-, control; ---, $1 \mathbf{a} ;-\bullet-, \mathbf{2 a} ;-\bullet-, \mathbf{3 a} ;-\mathbf{\Delta -}, \mathbf{4}$ (at $\mathrm{IC}_{25}$ conc.). The values are means of three separate experiments.

Therefore, we have considered of interest the use of ${ }^{1} \mathrm{H}$ NMR spectra for the identification and evaluation of the inhibiting effect caused by the compounds in metabolites excreted by T. cruzi. An example of the types of spectra obtained with Graces medium is shown in the different frames of Fig. (3I) displays the spectrum obtained with fresh (uninoculated) medium, and Fig. (3II) corresponds to the spectrum given by cell-free medium 4 days after inoculation with the T. cruzi strain. Additional peaks, corresponding to the major metabolites produced and excreted during growth, were detected when the last spectrum (Fig. 3II) was compared with that one obtained with fresh medium (Fig. 3I). The metabolites excreted were mainly acetate, succinate, and L-alanine. When the flagellates were treated with four compounds, the excretion of some of these catabolites was clearly inhibited, mainly in the cases of $\mathbf{4}$, which inhibited

\section{I}

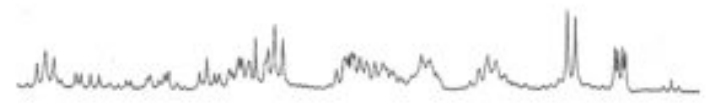

II
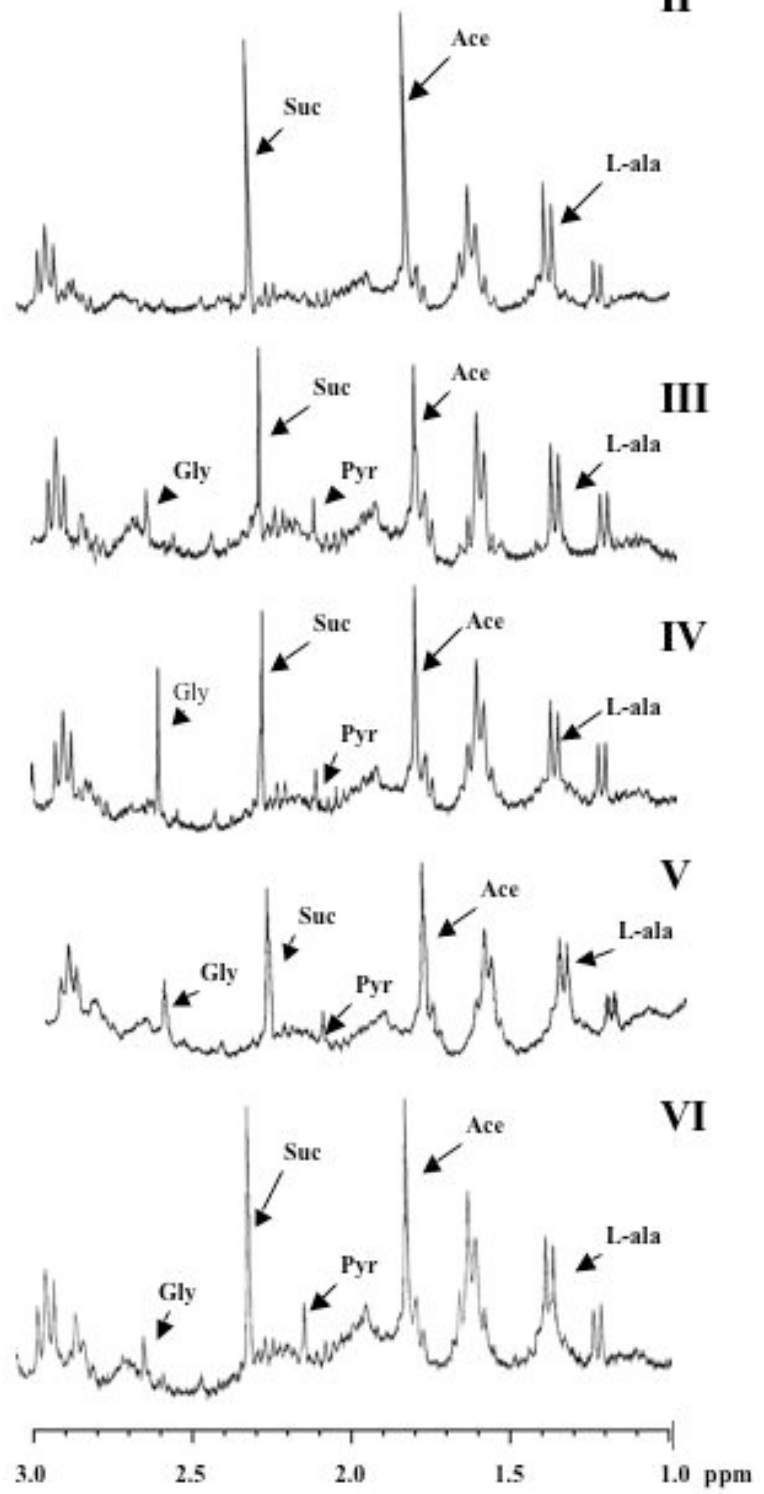

Fig. (3). ${ }^{1} \mathrm{H}-\mathrm{NMR}$ spectra epimastigote forms of $T$ cruzi treated against flavonoids compounds (at a concentration of $\mathrm{IC}_{25}$ ): (I) fresh medium, (II); Control (untreated); (III) 1a; (IV) 3a; (V) 4 and (VI) 2a. L-ala, alanine; Ace, acetate; Pyr, pyruvate; Suc, succinate and Gly, glycerol. 
mainly the production of acetate and succinate by 34 and 35 $\%$, respectively, at the dosage assayed $\left(\mathrm{IC}_{25}\right)$ (Fig. $\left.3 \mathrm{~V}\right)$. Meanwhile, 1a caused inhibitions of 32 and $20 \%$ in the production of these two metabolites (Fig. 3III) whereas 3a inhibited acetate and succinate production by 20 and $22 \%$, respectively, but pyruvate production increased significantly (Fig. 3IV), respectively, order of effectiveness in metabolite inhibition being $\mathbf{4}>\mathbf{1 a}>\mathbf{3 a}$. The effect on the excretion products could be due to the action that the compounds tested exerted on enzymes involved in their degradation pattern or to a loss in functionality of the mitochondrion, the organelle where succinate and acetate are formed by these parasites, since glycolysis is compartmentalized [14]. This hypothesis is reinforced by electron-microscope data. The main role of the production of these metabolites was to maintain the redox balance [20, 21], as its production was inhibited. Parasites develop alternative strategies to subsist, opening other fermentative pathways by which they produce L-alanine, ethanol, glycerol, and even pyruvate, thereby maintaining the redox balance.

Morphological alterations of $T$. cruzi epimastigote treated with compounds 1a, 2a, 3a and $\mathbf{4}$ were examined by transmission electron microscopy (TEM). All the compounds assayed at $\mathrm{IC}_{25}$ for $72 \mathrm{~h}$ induced lysis or alterations of the
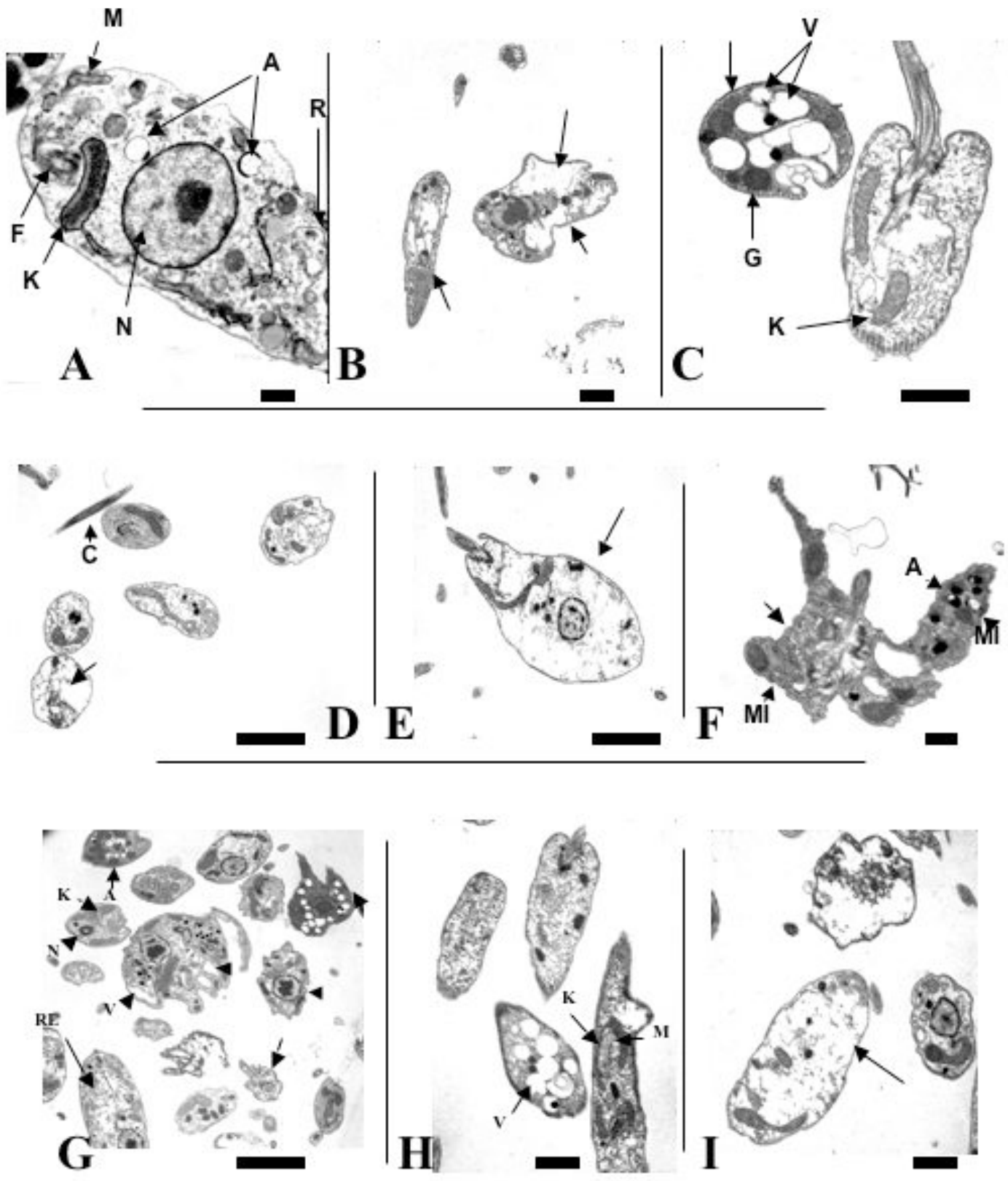

Fig. (4). Ultraestructural alterations by TEM in $T$. cruzi treated with acetylated flavonoids compounds (at a concentration of $\mathrm{IC}_{25}$ ). (A) Control parasite, $12000 \times($ bar= $0.583 \mu$ ). (B and C) Epimatigote forms of T. cruzi treated with 4, 12000 and $7000 \times$ (bar=0.583 and $1.00 \mu$ respectively). (D and E) Epimatigote forms treated with 2a, $4400 \mathrm{x}$ (bar=1.59 $\mu$ ). (F and G) Epimatigote forms treated with $1 \mathbf{a}, 4400 \mathrm{x}$

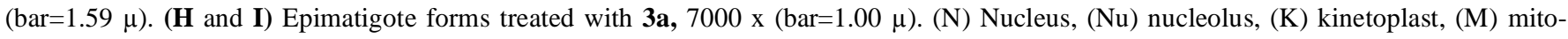
chondrion, (F) flagellum, (V) vacuoles, (G) glycosomes, (R) reservosomes, (C) cytoplasmic organelles, (Mi) myelin-like figures and (A) acidocalcisomes. 
parasites (Figs. 4-A to I) as compared to control cells (Fig. 4-A).

The treatment with 4 (Figs. 4-B and C) caused death in some parasites and distortion of the parasite body, swelling, and flattening. The compound shortened cell length and severely distorted cell shape (Fig. 4-B). Frequent cells alterations included the disruption of the cytoplasm with marked alterations of reservosomes (arrow heads, Figs. 4-B and C) and strong cytoplasmic vacuolisation. Glycosomes were abundant and swelling appeared in some cases (Fig. 4-C). The kinetoplast was also altered (Fig. 4-C), with distortion of the DNA helicoidal structure.

Parasites treated with 2a showed great alterations (Figs. 4-D and E). Most of the cells died at this concentration and many of cytoplasmic organelles appeared free in the culture (Fig. 4-D), by the rupture of the parasites. Epimastigotes were smaller and the distortion of the nucleus, cytoplasm, and plasma membrane was clear (Fig. 4-E). The treatment with 1a (Figs. 4-E and F) caused different alterations. Most of the parasites showed complete alteration in shape. Other parasites presented alterations in shape, becoming almost unrecognizable, with electrodense cytoplasm that hampered the identification of the cytoplasmic organelles (Figs. 4-F and $\mathbf{G})$. In these parasites the acidocalcisomes and endoplasmic reticulum were abundant (Fig. 4-F). Dead parasites were also detected. Nuclei could not be found in some cells and appeared disrupted in others (Fig. 4-G). Concentric structures, myelin-like figures, appeared in some parasites and vacuolization was frequent. The mitochondrion and kinetoplast were also affected by the treatment with 1a, (Fig. 4-G) appearing swollen. Glycosomes were normal in some parasites but in others appeared less electrodense.

The product 3a caused no alterations in the parasite shape but many cells were dead (Figs. 4-H and I). The most frequent alterations were the swelling of the mitochondria and disruption of kinetoplastic DNA (Fig. 4-H) together with the formation of autophagic vesicles and progressive vacuolization of the cytoplasm (Figs. $\mathbf{4 H}$ and I). Some parasites showed abundant endoplasmic reticulum or ribosomes in the cytoplasm (Fig. 4-H) while in other parasites the cytoplasm was almost empty, containing only the degraded remains of the nucleus and cytoplasmic organelles (Fig. 4-I).

This is the first study made on the trypanocide activity of aceylate flavonoid compounds obtained from $C$. oliveriana. Our results show that the compounds tested here were highly active in vitro against both the extracellular as well as the intracellular forms of $T$. cruzi. These compounds (mainly acetylated derivatives obtained in a natural way from the plant) are not toxic to the host cells and are effective at concentrations similar to or lower than the reference drug used in the present study. The in vitro growth rate of $T$. cruzi was depressed, its capacity to infect cells was diminished and the multiplication of the amastigotes was greatly reduced.

In conclusion, our study provides data that these flavonoid derivatives have promising anti-trypanocide properties. These could have implications for other intracellular pathogens or phylogenetically related parasites, as shown for Leishmania spp. The potent trypanocidal activities of the plant-derived flavons described here represent an exciting advance in the search for new antiprotozoal agents.

\section{ACKNOWLEDGEMENTS}

This investigation received financial supports from MEC (Spain) - CGL2006-27889-E and CGL2008-03687-E.

\section{REFERENCES}

[1] OPS/WHO/NTD/IDM. Conclusiones y Recomendaciones de la Reunión Anual Conjunta IPA-AMCHA. VII ${ }^{\mathrm{a}}$ Reunión Anual de la Iniciativa Andina de Control de la Enfermedad de Chagas (IPA) y la III ${ }^{\text {era }}$ Reunión Anual de la Iniciativa de Vigilancia y Prevención de la Enfermedad de Chagas en la Amazonía (AMCHA) OPS/HDM/CD/425-06, 2006.

[2] Schmunis, G. The globalization of chagas disease. ISBT Sci. Ser., 2007, 2, 6-11.

[3] Urbina, J.A. Chemotherapy of chagas Disease. Curr. Pharm. Des., 2002, 8, 287-295.

[4] Apt, W.; Arribada, A.; Zulantay, I. G.; Vargas, S.L.; Rodríguez, J. Itraconazole or allopurinol in the treatment of chronic American trypanosomiasis: the regression and prevention of electrocardiographic abnormalities during 9 years of follow-up. Ann. Trop. Med. Parasitol., 2003, 97, 23-29.

[5] Kayser, O.A.; Kiderlen, K.; Croft, S.L. Natural products as antiparasitic drugs. Parasitol. Res., 2003, 90(2), S55- S62.

[6] Kolak, U.; Oztürk, M.; Ozgökce, F.; Ulubelen, A. Norditerpene alkaloids from Delphinium linearilobum and antioxidant activity. Phytochemistry, 2006, 67(19), 2170-2175.

[7] Melnichuk, G.G. Consolida regalis flavonoids (Delphinium consolida). Inst Bot Kiev, USSR. Chem. Abstr., 1972, 7, 322-33.

[8] González, P.; Marín, C.; Rodríguez-González, I.; Hitos, A.B.; Rosales, M.J.; Reina, M.; Díaz, J.G.; González.Coloma, A.; Sánchez-Moreno, M. In vitro activity of C20 diterpenoid alkaloid derivatives in promastigotes and intracellular amastigotes of Leishmania infantum. Int. J. Antimicrob. Agents, 2004, 25, 136141.

[9] Tasdemir, D.; Kaiser, M.; Brun, R.; Yardley, V.; Schmicy, T.J.; Tosun, F.; Rüedi, P. Antitrypanosomal and antileishmanial activities of Flavonoids and their analogues: In vitro, in vivo, structureactivity relationship, and quantitative structure-activity relationship studies. Antimicrob. Agents. Chemother., 2006, 50(4), 1352-1364.

[10] Luque, F.; Femández-Ramos, C.; Entrala, E.; Rosales, M.J.; Navarro, J.A.; Romero, M. A.; Salas-Peregrín, J.M.; SánchezMoreno, M. In vitro evaluation of newly synthesised $[1,2,4]$ triazolo [1,5-a] pyrimidine derivatives against Trypanosoma cruzi, Leishmania donovani and Phytomonas staheli. Comp. Biochem. Physiol., 2000, 126, 39-44.

[11] Díaz, J.G.; Carmona, A.J; Torres, F.; Quintana, J.; Estévez, F.; Herz, W. Cytotoxic activities of flavonoid glycoside acetates from Consolida oliveriana. Planta Med., 2008, 74, 171-174.

[12] Osuna, A.; Adroher, F.J.; Lupiañez, J.A. Influence of electrolytes and non-electrolytes on growth and differentiation of Trypanosoma cruzi. Cell. Differ. Dev., 1990, 30, 89-95.

[13] Nakajima-Shimada, J.; Hirota, Y.; Auki, T. Inhibition of Trypanosoma cruzi growth in mammalian cells by purine and pirimidine analogs. Antimicrob. Agents. Chemother., 1996, 40, 2455-2458.

[14] Sánchez-Moreno, M.; Lasztity, D.; Coppens, I.; Opperdoes, F.R. Characterization of carbohydrate-metabolism and demonstration of glycosomes in a Phytomonas sp. isolated from Euphorbia characias. Mol. Biochem. Parasitol., 1992, 54, 185-200.

[15] Torres, F.; Quintana, J.; Díaz, J.G.; Carmona, A.J.; Estévez, F. Trifolin acetate-induced cell death in human cells is dependent ton caspase- 6 and activates the MAPK pathway. Apoptosis, 2008, 13, 716-728.

[16] Anthony, J.P.; Fyfe, L.; Smith, H. Plant active components - a resource for antiparasitic agents? Trends Parasitol., 2005, 21(10), 462-468.

[17] Fernandez-Becerra, C.; Sánchez-Moreno, M.; Osuna, A.; Opperdoes, F.R. Comparative aspects of energy metabolism in plant trypanosomatids. J. Eukaryot. Microbiol., 1997, 44, 523-529.

[18] Vickerman, K. The evolutionary expansion of the Trypanosomatid flagellates. Int. J. Parasitol., 1994, 24, 1317-1331.

[19] Fairlamb, A.H.; Opperdoes, F.R. in Carbohydrate Metabolism in Cultured Cells, Morgan. M. J.; Ed.; Plenum: New York, 1986, pp. 183-224.

[20] Tielens, A.G.; Van Hellemond, J.J. Differences in energy metabolism between Trypanosomatidae. Carbohydrate metabolism in cultured cells. Parasitol. Today., 1998, 14(7), 265-272. 
[21] Besteiro, S.; Biran, M.; Biteau, N.; Conotou, V.; Baltz, T.; Canioni, P.; Bringaud, F. Succinate secreted by Trypanosoma brucei is produced by a novel and unique glycosomal enzyme. NADH- dependent fumarate reductase. J. Biol. Chem., 2002, 277, 3800138012 .

(C) Boutaleb-Charki et al.; Licensee Bentham Open.

This is an open access article licensed under the terms of the Creative Commons Attribution Non-Commercial License (http://creativecommons.org/licenses/by-nc/3.0/) which permits unrestricted, non-commercial use, distribution and reproduction in any medium, provided the work is properly cited. 